\title{
A COMPARISON OF THE GROWTH PERFORMANCE BETWEEN CATTLE REARED IN CONVENTIONAL SYSTEMS AND IN FERAL CONDITIONS
}

Sergio Nogales ${ }^{a}$, Juan Calderón ${ }^{b}$, Teresa Marta Lupic ${ }^{c}$, Maria Cristina Bressan ${ }^{d}$, Juan Vicente Delgado $^{a}$, María Esperanza Camacho ${ }^{\mathrm{e}}$

a Departmento de Genética, Universidad de Córdoba, Campus de Rabanales, 14071 Córdoba, Spain

b Estación Biológica de Doñana (Consejo Superior de Investigaciones Científicas), Américo Vespucio s/n, 41092 Sevilla, Spain

c Escola Superior Agrária do Instituto Politécnico de Castelo Branco, 6001-909 Castelo Branco, Portugal

d Faculdade de Medicina Veterinária, Universidade Lusófona de Humanidades e Tecnologias, 1749-024 Lisboa, Portugal

e Instituto de Investigación y Formación Agraria y Pesquera (IFAPA), Alameda del Obispo, 14004 Córdoba, Spain

Keywords: Natural growth Non-linear models Biological growth curve Commercial growth curve ABSTRACT

Feral and conventional growth performances were compared using Marismeña cattle as a model. Marismeña calves are commonly reared under feral conditions in one of the most important reserves of Europe (Doñana National Park, Spain). Data recording in these natural conditions faces compromises as animals are only handled once per year. This fact has to be saved to obtain efficient estimations for the biological growth curve of cattle reared under feral conditions. On the one hand, we assessed the inference of the theoretical influence of human management on cattle growth. On the other hand, we studied the fitness of the best growth curve, in both feral and conventional systems to use the physiological meaning of the parameters obtained from their study as selection criteria related to the adaptability of potential breeding males and females. Fitting of Brody's, von Bertalanffy, Verhulst, logistic, Gompertz and Richards' models was tested as these models are the most representative ones for cattle growth. In general, Brody's and Richards' models presented the best fitting values for the biological curve. According to the biological curve parameters, males and females presented asymptotic weights of $641.71 \mathrm{~kg}$ and $403.55 \mathrm{~kg}$, respectively. As expected, the results of the commercial growth curve severely differed from those of the biological curve. The best fitting biological curve was not representative for cattle reared under commercial conditions. The logistic model was the best fitting one for feral females, Gompertz model for feral males, and Verhulst for intensive males and females, respectively. Seasonal oscillations in feeding may be responsible for the earlier achievement of the best performance in feral cattle ( 7 and 10 months for males and females, respectively), while such best performances were reached at 11 months in intensive calves, what becomes relevant for management and slaughtering decision-making. The study of the biological curve in Marismeña feral breed is very illustrative as this is the first time that feral cattle's growth is approached. Knowledge on the biological growth curve parameters could be used to interpret the strong relation between feral animals and their environment. This research could infer a model to quantify the effects of human management on livestock development, as feral resources offer unique opportunities to study domestic livestock without any human influence. 